Felix Rauh*

\title{
Memobase - Das Portal zum audiovisuellen Erbe der Schweiz
}

https://doi.org/10.1515/bfp-2020-2037

Zusammenfassung: Mit dem multimedialen Portal Memobase macht Memoriav, die Fachstelle für die Erhaltung des audiovisuellen Erbes der Schweiz, Bilder und Töne aus Gedächtnisinstitutionen sicht- und hörbar. Das Portal ermöglicht die Suche in Beständen aus mehr als 60 Institutionen aus allen Sprachregionen der Schweiz. Dazu gehören auch die bewegten Bilder der mehrsprachigen Schweizer Filmwochenschau oder Filme über die humanitären Aktionen des Internationalen Komitees vom Roten Kreuz.

Schlüsselwörter: Memobase; Memoriav; Film; Schweiz; Portal; Filmwochenschau

\section{Memobase-The Portal to Switzerland's Audio-visual Heritage}

Abstract: With the multimedia portal Memobase, Memoriav, the specialist agency for the preservation of Switzerland's audio-visual heritage, makes images and sounds from memory institutions visible and audible. The portal allows users to search in collections from more than 60 institutions from all language regions of Switzerland. It also includes films on the humanitarian actions of the International Committee of the Red Cross or the moving images of the multilingual Swiss newsreel.

Keywords: Memobase; Memoriav; films; Switzerland; portal; newsreel

\section{Einleitung}

In Gedächtnisinstitutionen finden sich große Mengen an bewegten Bildern unterschiedlichster Provenienz, die nur sehr selten auf DVD oder auf kommerziellen VideoStreaming-Plattformen publiziert werden. Dazu gehören Auftragsfilme, Amateurproduktionen, Fernsehsendungen, aber auch Spiel- und Dokumentarfilme, die in Vergessenheit geraten sind. Mittlerweile existieren im Internet viele Orte, wo solche Werke deponiert und mehr oder weniger gut dokumentiert visioniert werden können. $\mathrm{Zu}$ nennen ist

*Kontaktperson: Felix Rauh, felix.rauh@memoriav.ch neben den bekannten kommerziellen Portalen YouTube und Vimeo insbesondere die reichhaltige Webseite mit frei zugänglichen audiovisuellen Ressourcen von archive.org.

In der Schweiz ermöglicht das bereits seit 19 Jahren existierende Portal Memobase den Zugang zu archivierten Fotos, Ton- Film- und Videodokumenten. Es wird von Memoriav, der Fachstelle für die Erhaltung des audiovisuellen Kulturerbes der Schweiz, betrieben. Memoriav ${ }^{1}$ ist ein 1995 von Schweizer Kulturerbe-Institutionen gegründeter Verein, zu dessen Gründungsmitgliedern u. a. die Schweizerische Nationalbibliothek, das Schweizerische Bundesarchiv, das Schweizer Filmarchiv, die Schweizerische Nationalphonothek und das Bundesamt für Kommunikation gehören.

Seit 1995 unterstützt Memoriav mit Geld des Bundes Schweizer Gedächtnisinstitutionen bei der langfristigen Sicherung ihrer Foto-, Ton-, Film- und Videobestände. Dies geschieht durch die Vermittlung von Wissen und durch die Vergabe von Förderbeiträgen an Erhaltungsprojekte. Eine der Förderbedingungen ist die Zugänglichkeit der Dokumente für das Publikum zumindest in der Institution und die Publikation der Metadaten auf Memobase.

Die Präsentation der ersten Memobase fand ausgerechnet am 11. September 2001 im Bundesarchiv in Bern statt. An diesem Tag, der wegen der Terrorangriffe in den USA als 9/11 in die Geschichte einging, waren aufgrund der weltweiten massiven Internetzugriffe die Datenleitungen nicht in der Lage, eine Abfrage im Portal darzustellen. Auch mit funktionierendem Internet hätten keine bewegten Bilder gezeigt werden können. Die erste Generation der Memobase stellte Metadaten in Dublin Core aus Projekten, die von Memoriav gefördert wurden, zur Verfügung. Aus rechtlichen und technischen Gründen konnten die Bilder und Töne damals nur in seltenen Fällen direkt zugänglich gemacht werden. Die Treffer einer Suche verwiesen auf den Konsultationsort, wie den audiovisuellen Lesesaal des Schweizerischen Bundesarchivs. Dort konnten z. B. die Beiträge der Tagesschau des Schweizer Fernsehens, des wichtigsten televisuellen Informationsmediums, auf professionellen Videokassetten angeschaut und auf VHS kopiert werden.

1 https://memoriav.ch/. 

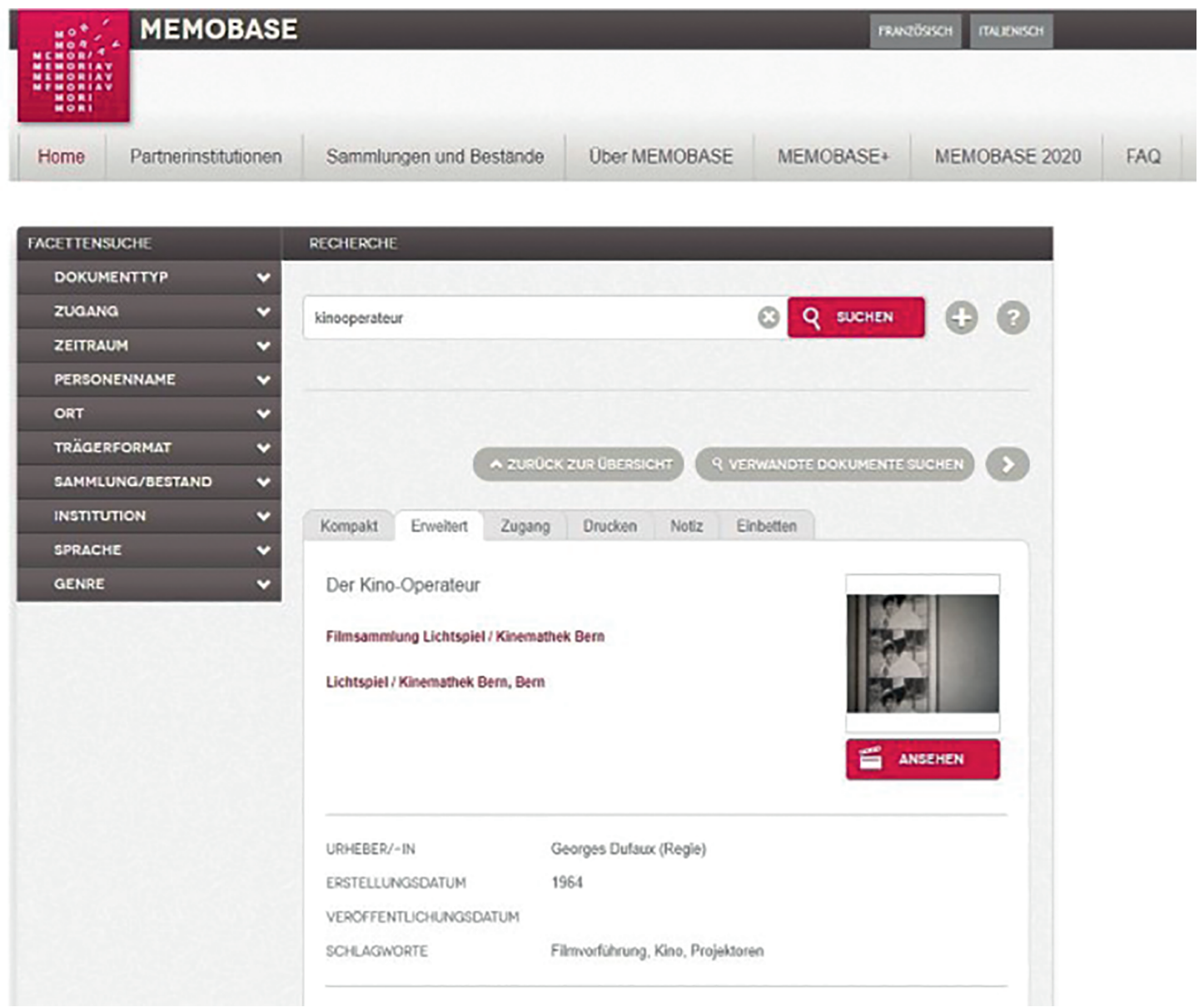

Abb. 1: Der Kino-Operateur. Film aus der Sammlung der Lichtspiel Kinemathek. Screenshot mit Metadaten aus Memobase ${ }^{2}$

In den letzten Jahren wurde Memobase mehrfach weiterentwickelt. Einige Grundsätze blieben aber gültig. Die Daten stammen immer noch in erster Linie aus den Memoriav-Förderprojekten. Da Memoriav sich um alle audiovisuellen Medien, inkl. Fotos kümmert, kommt auf Memobase ein reicher Mix an Medien und Themen aus Archiven, Bibliotheken, Museen, Radio- und Fernsehstationen der vier Sprachregionen zusammen. Um diese Heterogenität bewältigen zu können, werden auch in der aktuellen Portalversion alle Metadaten in ein einheitliches Datenmodell übernommen.

\section{Metadaten in Memobase}

Heute stellt Memobase Informationen zu den Dokumenten auf den drei Ebenen Dokument - Bestand - Institution zur

2 Film Der Kino-Operateur von George Dufaux aus dem LichtspielBestand: https://memobase.ch/de\#document/LS-176649 (aufgerufen am 29.9.2020).
Verfügung. Jedes Einzeldokument ist einem Bestand und dieser wiederum der Institution seiner Herkunft zugeordnet. Während die Präsentation der Institution kurzgehalten wird, orientiert sich die Beschreibung des Bestandes an archivischen Gepflogenheiten, d.h., die Lesenden lernen Menge und Umfang der Dokumente des Bestands, seine Überlieferungsgeschichte und allfällige Details zur Erschließung kennen. Sie erhalten damit Informationen, die für die quellenkritische Interpretation der Dokumente eines Bestandes essenziell sind. Die Informationen zu den einzelnen Bildern und Tönen sind heute nicht mehr in Dublin Core, sondern in eine Weiterentwicklung des Rundfunk-Standards EBU-Core dargestellt. Der Kerndatensatz genannt „Memobase-Core“ ist mit den internationalen Grundsätzen für die archivische Verzeichnung (ISAD -G: International Standard Archival Description -

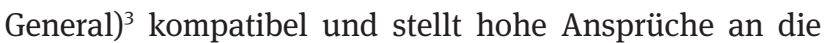
Qualität der deskriptiven und kontextuellen Metadaten.

3 https://www.ica.org/en/isadg-general-international-standard-arc hival-description-second-edition. 


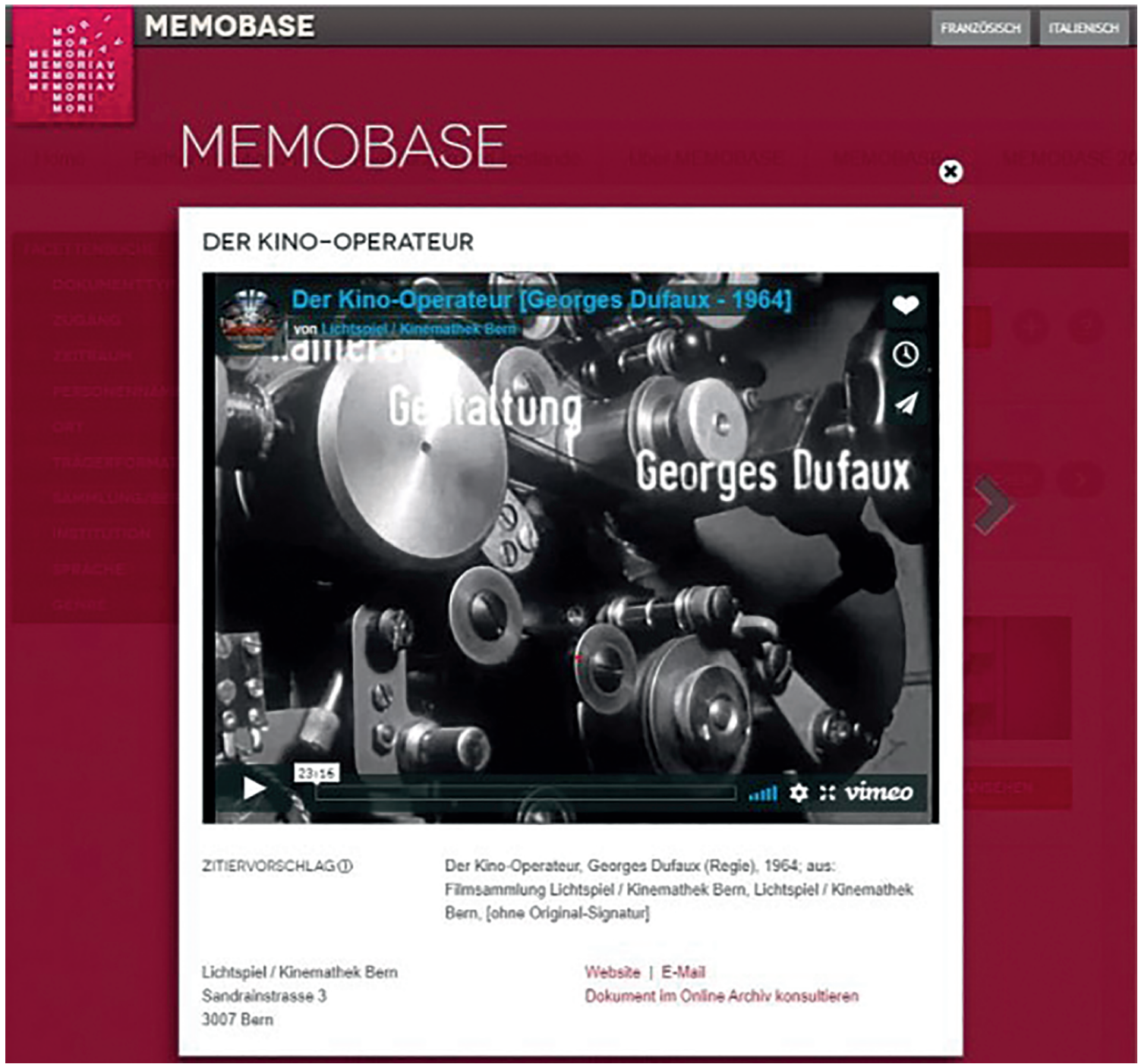

Abb. 2: Der Kino-Operateur. Screenshot Memobase-Video-Player

Damit hebt sich Memobase deutlich von anderen Plattformen ab, die selten genügend Metadaten für ein fundiertes Verständnis der gezeigten Inhalte bereitstellen. In der Regel erfolgt die Lieferung der Metadaten bei Projektende. In einigen Fällen können Daten direkt durch eine APISchnittstelle ausgetauscht und regelmäßig aktualisiert werden. Ansonsten sind die Datenlieferanten aufgefordert, Anpassung der Metadaten bei sich zu melden, damit sie auch in Memobase aktualisiert werden können.

\section{Zugang zu Bildern und Tönen}

Memoriav macht den Zugang zu den Bildern und Tönen in Memobase möglich. Dabei hält Memobase diese Dateien nicht selber, sondern bettet die auf anderen Infrastrukturen gespeicherten Dokumente in den Memobase-Player ein. Dieses Vorgehen funktioniert mit den großen kommerziellen Videoplattformen YouTube und Vimeo, ebenso wie mit SRF-PLAY, der Streaming-Plattform des öffentlichrechtlichen Deutschschweizer Radio und Fernsehens.

Zusätzlich offeriert Memoriav mit Memobase+ einen eingeschränkten Zugang an Hör- und Visionierstationen, die von der Schweizer Nationalphonothek ${ }^{4}$ betrieben werden. Dabei handelt es sich um Computer, die direkt mit den Servern des Tonarchivs in Lugano verbunden sind, ohne dass Manipulationen (Download etc.) möglich sind. Die Phonothek richtete diese Lösung ein, um Schweizer Tondokumente aus ihrem Archiv, in einem rechtlich geschützten Rahmen, außerhalb der Südschweiz zugänglich zu machen. Memobase darf dieselbe Infrastruktur mitbenützen.

4 https://www.fonoteca.ch/. 
Aktuell sind insbesondere viele Radio- und TV-Sendungen noch nicht Online über Memobase konsultierbar. Memoriav unterstützte die Archive des SRG (die öffentlich-rechtliche Schweizerische Radio- und Fernsehgesellschaft) viele Jahre bei der Erhaltung von ausgewählten Sendegefäßen, deren Metadaten bereits auf Memobase gefunden werden können, die aber noch nicht in den OnlineZugängen der Radio- und Fernsehstationen angekommen sind. Als Alternative verweist Memobase im Fall von Dokumenten aus dem Deutschschweizer Fernsehen SRF ${ }^{5}$ auf FARO-Web, den geschützten Zugang zum SRF-Archiv. Dieser wird in ausgewählten Gedächtnisinstitutionen wie dem Schweizerischen Sozialarchiv ${ }^{6}$ in Zürich ermöglicht.

Alle anderen verzeichneten Bilder und Töne können vor Ort in der Institution angesehen oder angehört werden.

NutzerInnen haben die Möglichkeit, entweder einfach - über alle Felder - oder erweitert - auf gewisse Felder beschränkt - zu suchen. Außerdem bietet Memobase die Filterung des Suchresultats mithilfe von zehn Facetten. Dort kann u.a. auf Dokumententyp, Art des Zugangs, Zeitraum, Bestand und Institution eingeschränkt werden.

\section{Audiovisuelle Inhalte auf Memobase}

Mitte September 2020 verzeichnet Memobase annähernd eine halbe Million (414227) Dokumente aus 66 verschiedenen Schweizer Institutionen. Mehr als 50000 bewegte Bilder können online visioniert werden, darunter sind etwas mehr als die Hälfte Fernsehbeiträge und mehr als 20000 Filmdokumente. Auf drei Filmbestände wird im Folgenden näher eingegangen.

\subsection{Beispiel: Lichtspiel Kinemathek Bern}

Von der Lichtspiel Kinemathek ${ }^{7}$ in Bern sind aktuell zwar erst 57 von 6876 beschriebenen Filmen online. Da sich diese Zahl in den nächsten Monaten stark erhöhen wird, lohnt sich der regelmäßige Blick in die Sammlung. Die Berner Institution etablierte sich in den letzten Jahren als zweites professionelles Schweizer Filmarchiv neben der Cinémathèque suisse. ${ }^{8}$ Die auf Memobase verzeichneten Lichtspiel-Filme gehören zu ganz verschiedenen Genres,

5 https://www.srf.ch/.

6 https://www.sozialarchiv.ch/.

7 https://lichtspiel.ch/de/.

$8 \mathrm{https} / / /$ www.cinematheque.ch/d/. darunter fallen die vielen Amateurproduktionen sowie Trick- und Musikfilme auf. Online verfügbar sind bisher am meisten dokumentarische Produktionen, etwa Auftragsfilme für Industrie und Landwirtschaft oder solche, die sich mit dem Filmwesen beschäftigen. So erklärt Der Kino-Operateur (1964) von Georges Dufaux in 23 Minuten, wie das Handwerk des Filmvorführens vor der Einführung digitaler Projektionen funktionierte (siehe Abb. 1 und 2). Ganz anders gelagert sind einige Studienfilme aus dem Bereich Animation der Hochschule Luzern. Sie entstanden im letzten Jahrzehnt und zeigen die Innovationskraft von jungen Schweizer Filmschaffenden.

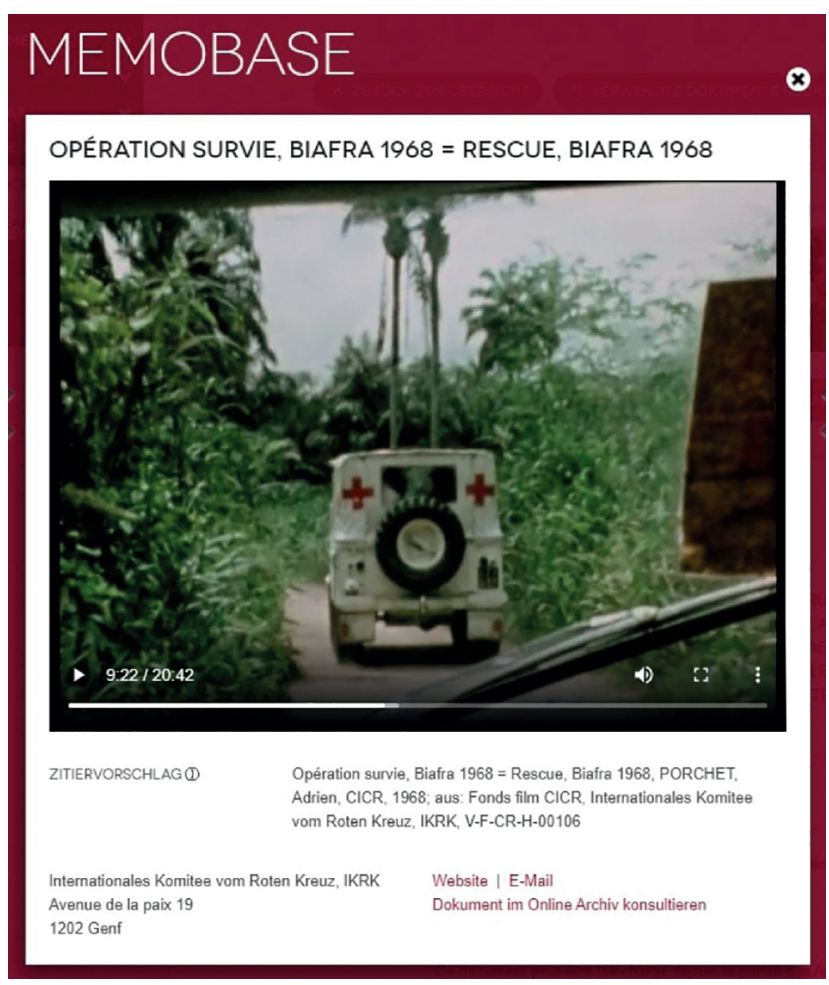

Abb. 3: IKRK-Film Opération Survie, Biafra 1968, Screenshot Memobase Video-Player ${ }^{9}$

\subsection{Beispiel: Internationales Komitee vom Roten Kreuz}

Auch das Internationale Komitee vom Roten Kreuz IKRK verfügt über ein sehr spannendes und bestens aufgearbeitetes Filmarchiv. ${ }^{10}$ Aufgrund einer engen Erhaltungs-

9 Film Opérations survie, Biafra 1968 von Adrien Porchet aus dem IKRK-Bestand: http://memobase.ch/de\#document/ICRC-5525. 10 Archives audiovisuelles du CICR. Webseite (in Französisch und Englisch): https://avarchives.icrc.org/. 
zusammenarbeit mit Memoriav können heute 119 Filme zur Arbeit der humanitären Organisation auf Memobase recherchiert und angeschaut werden. Speziell hervorzuheben sind Dokumentationen aus den 1920er-Jahren, welche die Repatriierung von hungernden russischen Kriegsflüchtlingen von der Krim nach Istanbul dokumentieren. Die Sammlung verfügt über eine breite Palette an Themen, welche die praktische Arbeit des IKRK in den Genfer Büros, aber auch auf dem Feld dokumentiert. Der französisch gesprochen Le Comité internationale de la Croix Rouge à Genève von 1942 zeigt die Arbeit der Zentralagentur für Kriegsgefangene, die Briefe von Angehörigen an gefangene Soldaten weiterleitet. Gleich sechs Filme dokumentieren die IKRK-Tätigkeit in Biafra. Der Gliedstaat wollte sich Ende der 1960er-Jahre von Nigeria lossagen, was zu einem blutigen Krieg führte. Im medialen Gedächtnis sind insbesondere die Bilder von hungernden Kindern hängen geblieben. Sie können in mehreren Filmen des Schweizer Dokumentarfilmers Adrien Porchet von 1968 über die Tätigkeit des IRKR im Kriegsgebiet verifiziert werden (siehe Abb. 3). Wer sich für Kriegschirurgie interessiert, kann sich War surgery: an introduction von 1988 anschauen. Der englisch kommentierte medizinische Lehrfilm erklärt die Behandlung von häufigen Verletzungen im afghanischen Kriegsgebiet. Er macht selten gezeigte Bilder öffentlich, die für nicht Einweihte schwer verdaulich sein können.

\subsection{Beispiel: Schweizer Filmwochenschau}

Als letzten sei ausführlich auf den Bestand der Schweizer Filmwochenschau (SFW) eingegangen, der vollständig auf Memobase visioniert werden kann. Die Schweizer Filmwochenschau wurde zwischen 1940 und 1975 in den Kinos der Schweiz gezeigt. Ihre in Deutsch, Französisch und Italienisch produzierten 1651 Ausgaben mit mehr als 6000 Beiträgen pro Sprache zeichnet ein vielseitiges Porträt der Schweiz. Im Kontext der sog. Geistigen Landesverteidigung entstanden Beiträge mit dem Ziel, eine spezifisch helvetische Eigenart zu kultivieren, und diente die SFW dazu, während des Zweiten Weltkrieges den Nazismus und den Faschismus und im Kalten Krieg den Kommunismus abzuwehren. Die in der Regel kurzen fünf bis sieben Beiträge pro Ausgabe zeichneten ein harmonisches Bild mit zufriedenen Menschen aus allen Landesteilen und Sprachregionen. Kritische Voten waren bis Ende der 1960er-Jahre kaum zu hören. Politische Themen standen selten im Vordergrund. Häufig zeigte die SFW gelebte Traditionen wie Volksfeste, kulturelle Anlässe und viel Sport, vorzugsweise aus dem Inland (siehe Abb. 4).

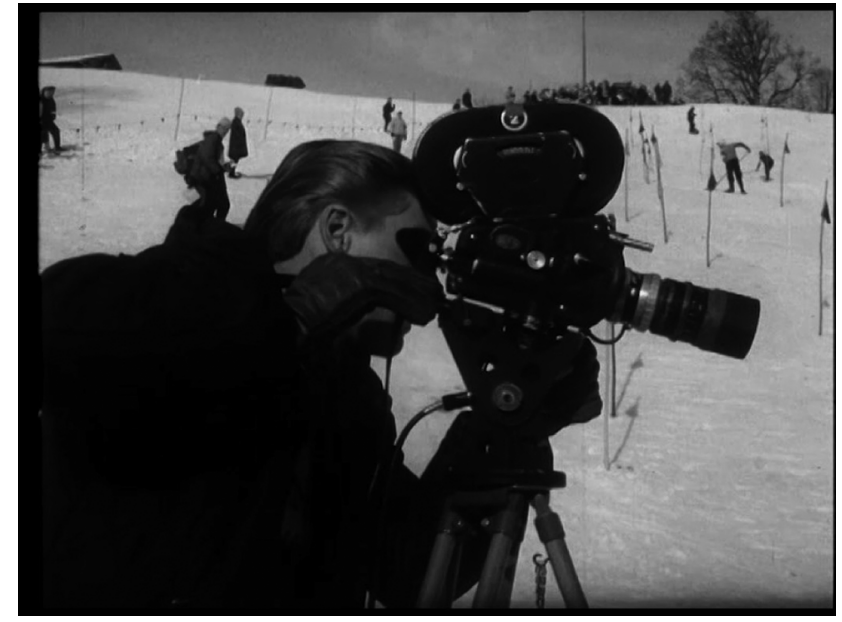

Abb. 4: Ein Kameramann der Schweizer Filmwochenschau am Skirennen in Grindelwald, 19.1.1962 ${ }^{11}$

Die Publikation der SFW auf der Memobase - und parallel dazu auch auf dem Online-Zugang des Schweizerischen Bundesarchivs - wurde nur möglich dank einer guten $\mathrm{Zu}$ sammenarbeit von Memoriav mit dem Bundesarchiv, der Cinémathèque suisse und dem Schweizer Fernsehen. Die beiden Gedächtnisinstitutionen und die TV-Anstalt steuerten die Filme und Textdokumente bei, Memoriav organisierte die Aufbereitung der Metadaten und die Integration in Memobase.

Die Projektpartner beschlossen der Schweizer Mehrsprachigkeit Rechnung zu tragen und die nicht mehr vorhandenen Sprachversionen zu untertiteln. Damit können alle Filmwochenschauen in den Sprachen Deutsch, Französische und Italienisch recherchiert und angeschaut werden. Die Untertitelung betrifft v. a. die italienische Sprachversion, deren Nitratkopien (1940-1952) fast vollständig vernichtet wurden. Auch eine große Zahl an französischsprachigen Kopien fehlt, wogegen die meisten deutschsprachigen überlebt haben. Über diese Besonderheiten, die für die Interpretation der Dokumente besonders wichtig sind, informiert die Bestandsbeschreibung, die aus jedem Beitrag direkt angewählt werden kann.

Wie bereits betont legt Memoriav besonderen Wert auf die gute Qualität v.a. der deskriptiven Metadaten, die zum einen die Suche erleichtern und zum andern auch ein besseres Verständnis der Bilder und Töne schafft. Im Fall der SFW konnte sowohl auf die dokumentarische Arbeit von SRF zurückgegriffen werden als auch auf sog. Communiqués, die mit den Filmen ausgeliefert wurden. Diese enthalten kurze Inhaltsbeschreibungen jedes Beitrags einer Ausgabe sowie die Transkription des Kommentars in allen

11 Quelle: Schweizerisches Bundesarchiv, Cinémathèque suisse. 
drei Sprachen. Sie sind damit eine äußerst wertvolle Informationsquelle. Für Memobase wurden sie gescannt, mit Texterkennungssoftware behandelt und anschließend von Studierenden korrigiert. Wer in Memobase die deutschsprachige SFW durchsucht, kann sich auf Sach- und Ortsdeskriptoren, indexierte Personennamen, Abstract und Sequenz-, d.h. Bildbeschreibung, sowie die Inhalte der Communiqués stützen (siehe Abb. 5). In den anderen beiden Sprachversionen musste auf die Übersetzung von Abstract und Sequenzbeschreibung des SRF aus Kostengründen verzichtet werden.

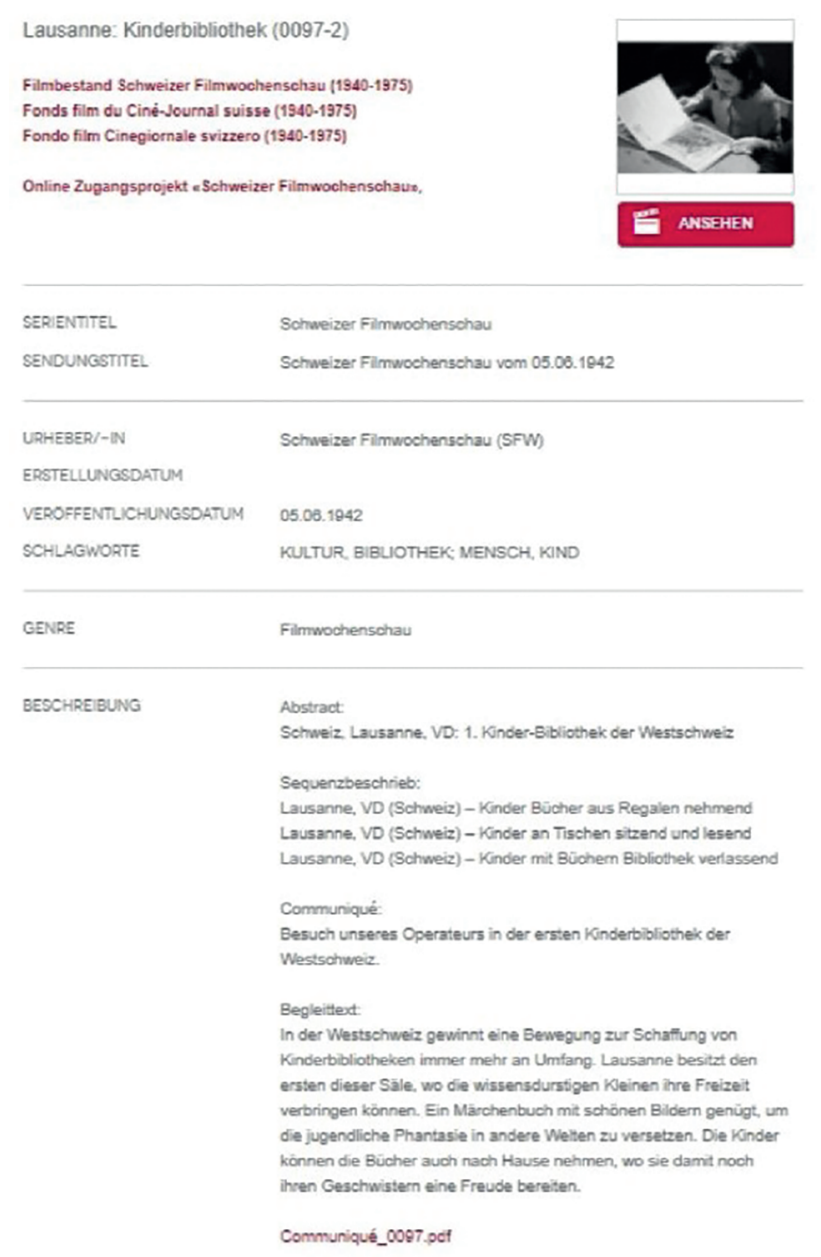

Abb. 5: Vielseitige deskriptive Metadaten. Beispiel Schweizer Filmwochenschau vom 5.6.1942 über eine Kinderbibliothek in Lausanne ${ }^{12}$

Im Fenster, das sich beim Drücken auf den roten Balken „Ansehen“ öffnet, kann der Beitrag visioniert werden. Der

12 https://www.memobase.ch/\#document/SFW_CJS_CGS-SFW_009 7-2.
Cursor springt direkt an die gewünschte Stelle. Wer sich für die anderen Inhalte derselben Woche interessiert, kann sich gleich die ganze Ausgabe ansehen. Und wer in diesem Fall mehr über diese anderen Sujets erfahren möchte, klickt in den Metadaten auf einen Link, der eine Liste mit allen Beiträgen generiert. Dasselbe ist auch möglich, wenn sich jemand für die anderen beiden Sprachversionen interessiert, die am gleichen Datum in den Kinos der italienisch- oder französischsprachigen Schweiz liefen.

\section{Ausblick}

Die Memobase bietet bereits in der aktuellen Version $\mathrm{Zu}$ gang zu zahlreichen Dokumenten des Schweizer audiovisuellen Kulturerbes. Mit dem Projekt Memobase 2020, ${ }^{13}$ das Memoriav seit 2019 zusammen mit der Universitätsbibliothek Basel realisiert, wird nicht nur die Systemarchitektur erneuert, sondern auch der Anspruch erweitert. Die Memobase der Zukunft möchte Aggregator für das ganze audiovisuelle Kulturerbe der Schweiz sein und deshalb auch Sammlungen von Institutionen aufnehmen, die keine Fördergelder von Memoriav erhalten haben. Am Qualitätsanspruch an die Metadaten ändert sich nichts. Im Gegenteil. Künftig wird wenn immer möglich auf Normdaten wie GND oder Wikidata zurückgegriffen. Als zusätzliche Attraktion ist geplant, in Zusammenarbeit mit externen Partnern sog. Vitrinen zur Verfügung zu stellen. Damit sind kuratierte Bild- und Tonauswahlen gemeint, die z.B. von Personen aus Forschung, Lehre und Bildung zu einem bestimmten Thema auf der Memobase zusammengetragen und kommentiert werden. Filminteressierte dürften also schon bald kostenlos auf noch mehr, attraktiv präsentierte, bewegte Bilder zugreifen können.

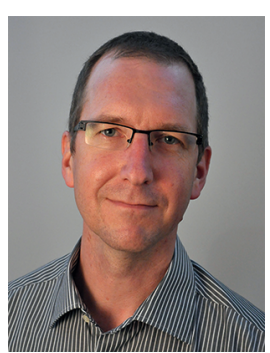

Felix Rauh

Bümplizstrasse 192

CH-3018 Bern

Schweiz

felix.rauh@memoriav.ch
13 Medienmitteilung zum Startschuss von Memobase 2020: https:// memoriav.ch/memobase2020/. 